\title{
Effects of flow disturbance on an ultrasonic gas flowmeter
}

\author{
E. HÅKANSSON* and J. DELSING*
}

A series of tests are carried out to assess the effects of flow disturbance on a small dimension ultrasonic gas flowmeter. Flow disturbances generated by cone couplings, and single and double elbows are investigated. Measurements with a $100 \mathrm{D}$ straight pipe upstream with a smooth connection to the meter body are used as a reference. Our measurements show that the symmetrical disturbance produced by a cone coupling at a $12 \mathrm{D}$ distance from the transducer path does not impair the performance of the flowmeter. An asymmetrical disturbance, such as a single or a double elbow at the same distance, seems generally to give an underestimation of the flow velocity, resulting in reading errors of $-1 \%$ or worse. Measurements with straight pipes of $10 \mathrm{D}, 20 \mathrm{D}, 40 \mathrm{D}$ and $80 \mathrm{D}$ between the disturbance and the flowmeter have also been made showing that $10 \mathrm{D}$ can cause an overestimation of flow velocity. Increasing the length of the straight pipe generally decreases the error. More than $80 \mathrm{D}$ straight pipe between the disturbance and the flowmeter is required to give a result within $\pm 1 \%$ of reference conditions. The angle between the elbow plane and the transducer plane is changed from 0 to $315^{\circ}$ in $45^{\circ}$ steps. The meter error is plotted as a function of inlet angle, showing a clear relationship between these values.

Keywords: flow disturbance; gas flowmeters; flowmeter geometry

\section{Introduction}

The accuracy of an ultrasonic gas flowmeter is dependent on the velocity distribution of the fluid between the two ultrasonic transducers. Flow disturbances are mostly generated by installation conditions, e.g. pipe connections, elbows, pumps or valves in front of the meter. These flow disturbances introduce errors in the flowmeter reading often called 'installation effects'. For ultrasonic flowmeters there are two major sources of error: area averaging and distorted sound.

Several authors have investigated installation effects in ultrasonic flowmeters. Höjholt ${ }^{1}$ has investigated the effects of flow disturbances on an ultrasonic fluid flowmeter. He claims that a $40 \mathrm{D}$ straight pipe in front of the meter will as a rule be enough to establish a good meter performance for an axially disturbed flow profile, but for a rotational flow profile (swirl) an $80 D$ straight pipe seems to be insufficient. Heritage ${ }^{2}$ has shown that a single or a double elbow in front of commercial ultrasonic flowmeters can lead to errors between $-1.5 \%$ and $-11 \%$. Halttunen and Luntta ${ }^{3-5}$ observe that every flowmeter type seems to be individual since measurements and simulations show that the

\footnotetext{
* Department of Heat and Power Engineering, Lund Institute of Technology, PO Box 118, Lund, Sweden, S-22100
}

same disturbance causes different errors in different meter types.

To reduce installation effects, manufacturers specify installation requirements. Mattingly and Yeh $^{6}$ have investigated the effects of swirl on some specific types of flowmeter showing that installation specifications are not always sufficient to establish good metering performance.

All the investigations mentioned above were made using liquid flowmeters. There have not been many investigations made on the effects of flow disturbances on ultrasonic gas flowmeters. However, the present work assesses the performance of an ultrasonic gas flowmeter developed at the Department of Heat and Power Engineering at Lund Institute of Technology ${ }^{7-9}$. The meter was tested in dry air under four different flow conditions:

A $100 \mathrm{D}$ straight pipe in front of the meter (reference condition)

$\square$ A cone coupling at a $12 D$ distance from the transducer path

$\square$ A single elbow in front of the cone coupling

$\square$ A double elbow out of plane in front of the cone coupling.

Our measurements show that the symmetrical disturbance produced by the cone coupling does not impair the performance of the flowmeter. An 
asymmetrical disturbance, such as the single or the double elbow, generally causes underestimation of the flow velocity, resulting in reading errors of $-1 \%$ or worse. Further, the orientation of the sound path with respect to the single or double elbow can greatly affect the errors.

\section{Experimental set-up}

\section{The sing-around gas flowmeter}

For the following discussion we give a brief review of the sing-around method as background. We will assume a configuration as shown in Figure 1. A sing-around loop is started when we transmit a short ultrasound pulse from, say, transducer I in the upstream direction. This pulse is received by transducer II and fed to the singaround electronics, which detect it and immediately start the transmission of the next ultrasound pulse in the same direction. This establishes a 'sing-around' loop which will be maintained for a predetermined number of loops. The same procedure is subsequently repeated in the downstream direction.

The sing-around loop will oscillate with a certain period $t$, called the 'sing-around period'. The singaround period depends on the speed of sound $c$, the medium between the transducers, the transducer distance $L$, and the fluid velocity $v$. Thus, we can write the downstream and upstream sing-around periods, $t_{1}$ and $t_{2}$, as:

$$
\begin{aligned}
& t_{1}=\frac{L}{(c+v) \cos \alpha} \\
& t_{2}=\frac{L}{(c-v) \cos \alpha}
\end{aligned}
$$

From the sing-around periods, $t_{1}$ and $t_{2}$, the fluid velocity $v$ is found as:

$$
v=\frac{L}{2 \cos \alpha}\left(\frac{1}{t_{1}}-\frac{1}{t_{2}}\right)
$$

To determine the fluid velocity we only need to know the transducer distance $L$, the interrogation angle $\alpha$, and the upstream and downstream sing-around periods, $t_{1}$ and $t_{2}$.

The flowmeter body (Figure 2) was made of Plexiglas in order to make visual inspection possible. The cone couplings and pipes used are all made of brass and copper, respectively. The cone couplings (shown at the ends of the flowmeter in Figure 2) are there to provide easy connections with the pipeline

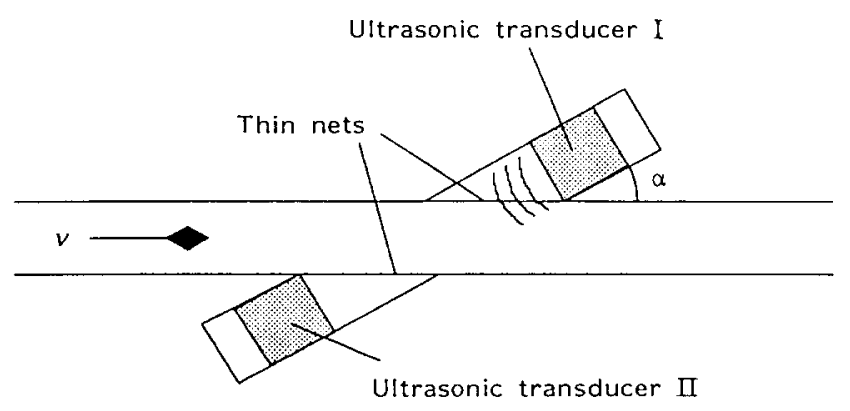

Figure 1 The sing-around flowmeter body

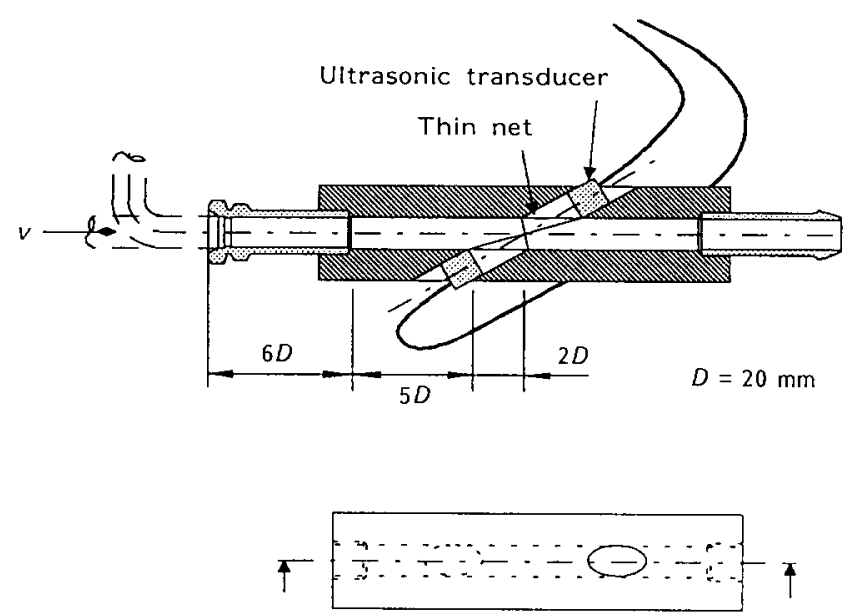

Figure 2 The flowmeter body is made of Plexiglas with cone couplings in brass/copper

which has the same internal diameter as the flowmeter. The internal surface of the couplings is not perfectly smooth but, as we show, any slight disturbance of the flow produced by the couplings does not affect the ultrasonic flowmeter measurement.

The distance between the transducers is $88 \mathrm{~mm}$ and, since the interrogation angle is $30^{\circ}$, the effective distance of gas and sound interaction is $76 \mathrm{~mm}$. The ultrasonic frequency used is $535 \mathrm{kHz}$ and the sound path diameter is $12 \mathrm{~mm}$. The pipe inside diameter is $20 \mathrm{~mm}$.

Unfortunately the design of the ultrasonic flowmeter body introduces volumes of gas in front of the transducers outside the main flow. These volumes cause heavy turbulence which impair the sound transmission through the pipe. Due to very high velocity fluctuations in front of the transducers the ultrasonic pulse is simply 'blown away'. In order to smooth this turbulence thin nets are placed in front of the transducers. These nets simulate the pipe wall for the gas flow but are transparent for the ultrasound.

Zero flow stability is one of the most important requirements for a large dynamic range. The zero flow stability for our meter was found to be $\pm 0.6 \mathrm{~mm} \mathrm{~s}^{-1}$ (Figure 3 ). The repeatability of the meter was $\pm 0.5 \%$

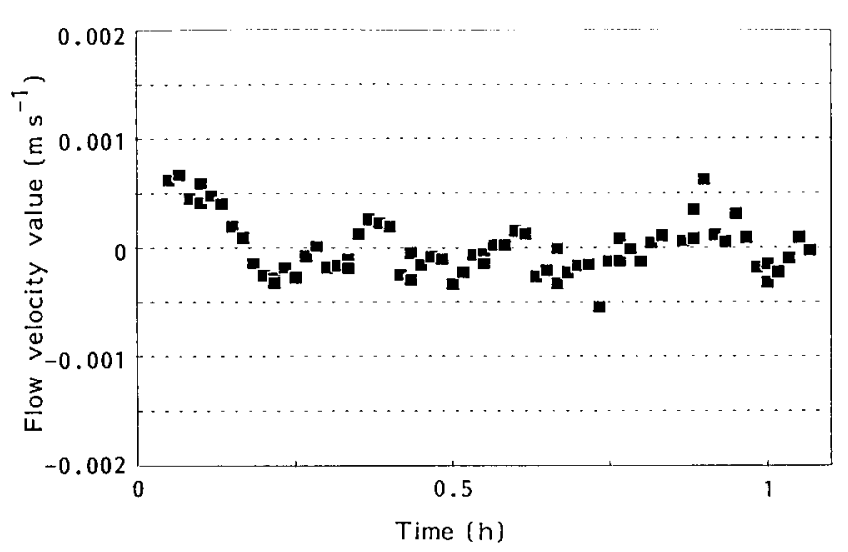

Figure 3 Zero flow stability of the ultrasonic gas flowmeter 
of measured flow velocity ${ }^{10}$. The reference meter used was a calibrated turbine gas flowmeter.

\section{The test rig}

The calibration rig mainly uses standard components, originally meant for use in compressor systems or similar (Figure 4). A central compressor system generates an airflow which is dried and then fed through a $10 \mu \mathrm{m}$ dust filter. After filtering, the air pressure is regulated to give an airflow as constant as possible. A voltage controlled pneumatic valve is used to adjust the flow velocity. Unfortunately this valve shows both hysteresis and non-linearity and together with variations in the incoming air pressure this leads to problems in keeping a constant airflow during a whole measuring sequence.

The piping in front of, and behind, the ultrasonic flowmeter is made of standard $20 \mathrm{~mm}$ copper pipe connected with brass cone couplings. The use of the cone couplings between the different parts leads to high flexibility.

Since the ultrasonic gas flowmeter measures the velocity of air passing through the pipe, a reference meter primarily sensitive to the same quantity is to be preferred. A turbine gas flowmeter, FT8-8AEE1-GEA-1 from Flow Technology Inc. was chosen ${ }^{11}$. In this meter the flow sensitive element is a freely suspended bladed rotor positioned axially in the flow stream. The rotational speed of the turbine is proportional to the velocity of the fluid. The rotation of the turbine rotor generates electrical pulses in the pickup which is attached to the flowmeter housing in close proximity to the turning rotor. These pulses are transformed into a square wave signal where frequency is proportional to the flow velocity. The actual flow velocity is then calculated by the control computer using linear interpolation between the two nearest values in the calibration data table supplied.

The reference flowmeter was calibrated in dry air for an extended range, $1: 20$, and according to the manufacturer the meter accuracy over the range of our experiments is within $\pm 1 \%$ of rate with a repeatability of $0.1 \%$ of rate. Due to restrictions set by the calibration rig the usable dynamic range of the reference meter is decreased to approximately $1: 12$. Correction is made for the non-linear reference meter characteristic. The reference meter is mounted with $50 \mathrm{D}$ straight pipe in front of it and behind it. The pipe diameter used is $10 \mathrm{~mm}$. This well exceeds the installation recommendations given by the manufacturer.

The calibration rig is controlled by an IBM PS/2 computer. In addition to data acquisition the computer

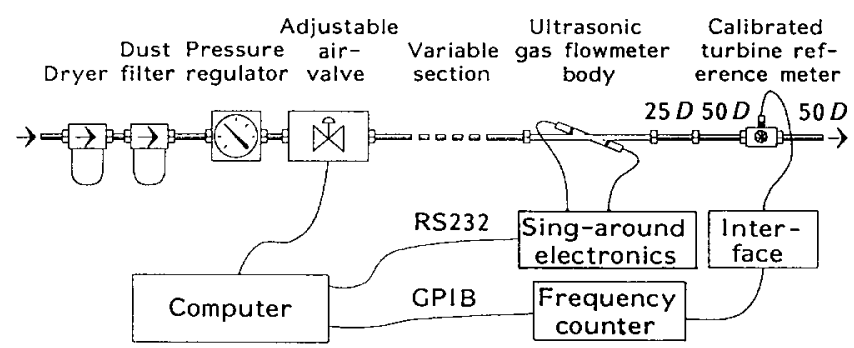

Figure 4 Schematic diagram of the calibration rig is used for processing and saving the data. The control computer uses the LabWindows environment in addition to an MIO-16 data acquisition card from National Instruments. The combination allows both controlling and measuring in addition to advanced analysis possibilities. The control computer communicates with the ultrasonic gas flowmeter via an RS232 link and the frequency meter, reading the reference meter value, is connected via GPIB.

To investigate the stability of the actual airflow, all measured flow velocity values in the same measuring cycle were plotted in a so called Youden plot ${ }^{12}$. The value measured by the reference meter gives the $x$ coordinate of a point marked and the $y$-coordinate is given by the corresponding ultrasonic flowmeter reading. The graph is divided into four quadrants by two median lines. If only random errors are present the measuring points are expected to be equally numerous in all quadrants.

The Youden plots reveal a variation of the actual flow velocity of up to $\pm 5 \%$ (Figure 5 ).

\section{Measurements}

The performance of our ultrasonic gas flowmeter was investigated under four different flow conditions:

Reference condition. A 100 D straight pipe mounted directly in front of the flowmeter body using a smooth connection (Figure 6a).

$\square$ A cone coupling placed in front of the meter body at a $12 D$ distance from the ultrasonic transducer path (Figure 6b).

$\square$ A single elbow made of a commercial $90^{\circ}$ copper bend with a centreline curvature of $1.5 \mathrm{D}$ connected upstream of the cone coupling (Figure $6 \mathrm{c}$ ).

$\square$ A double elbow out of plane made of two single elbows mounted perpendicular to each other connected upstream of the cone coupling (Figure $6 d)$.

In the last two $10 \mathrm{D}, 20 \mathrm{D}, 40 \mathrm{D}$ and $80 \mathrm{D}$ of straight pipe were introduced between the elbows and the flowmeter to determine proper installation requirements. Further, the sound path orientation with respect to the single and double elbow was varied.
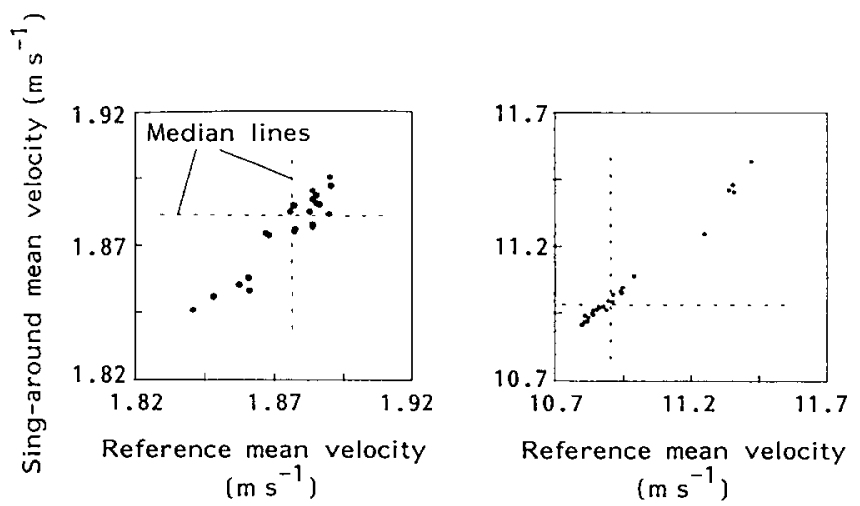

Figure 5 Youden plots for mean velocities of $1.9 \mathrm{~m} \mathrm{~s}^{-1}$ and $11 \mathrm{~m} \mathrm{~s}^{-1}$. Both clearly reveal a variation of the actual flow velocity during the measuring sequence 

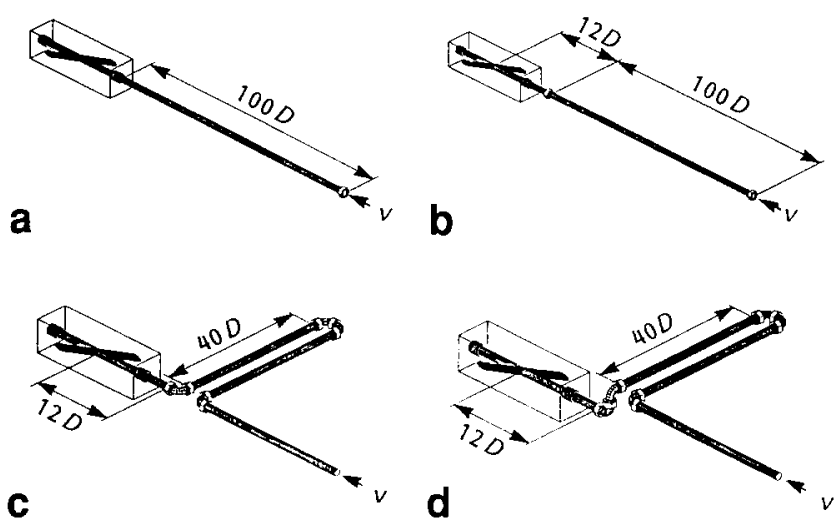

Figure 6 Four different flow conditions were investigated: (a) reference condition; (b) cone coupling; (c) single elbow; (d) double elbow out of plane

The angle between the transducer plane and the elbow plane is defined as the 'inlet angle'. It was changed from 0 to $315^{\circ}$ in steps of $45^{\circ}$.

\section{Data processing}

One reading from the ultrasonic gas flowmeter consists of a flow velocity value calculated as an average of 25 velocity values calculated using equation (3). This results in a sample frequency of $\sim 1 \mathrm{~Hz}$. The flowmeter reading also includes the standard deviation $\sigma_{1}$ in transit times, which is used as an indicator of the degree of turbulence between the transducers; normally $\sigma_{1}$ is less than a few percent.

To make comparison between the ultrasonic meter and the turbine meter feasible a mean of 20 flow velocity readings from the ultrasonic flowmeter was calculated. The standard deviation obtained here, $\boldsymbol{\sigma}_{2}$, was used as a quality factor for checking the stability of the air flow during one sequence. Under normal circumstances $\sigma_{2}$ is less than $1 \%$ of the measured flow velocity.

The error for the ultrasonic flowmeter is calculated according to equation (4).

$$
\text { Error }=100 \cdot \frac{\left(v_{\mathrm{us}}-v_{\mathrm{ref}}\right)}{v_{\text {ref }}}(\%)
$$

where $v_{\mathrm{us}}=$ the ultrasonic flowmeter reading $\left(\mathrm{m} \mathrm{s}^{-1}\right)$ and $v_{\text {ref }}=$ the reading of the reference meter $\left(\mathrm{m} \mathrm{s}^{-1}\right)$.

The error is plotted versus the estimated Reynolds number calculated as:

$$
\operatorname{Re}=\frac{\rho \cdot d \cdot v_{\mathrm{ref}}}{\eta} \approx \frac{1.1881 \cdot 0.02 \cdot v_{\mathrm{ref}}}{17.98 \cdot 10^{-6}}
$$

where $\rho=$ air density at $20^{\circ} \mathrm{C}\left(\mathrm{kg} \mathrm{m}^{-3}\right), d=$ pipe inside diameter $(\mathrm{m})$ and $\eta=$ the dynamic viscosity of

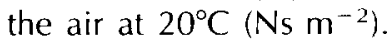

\section{Results}

The following diagrams present the ultrasonic flowmeter error plotted against Reynolds number determined by the reference meter. Figures 7 to 10 also include the two standard deviation values, $\sigma_{1}$ and $\sigma_{2}$. The left $y$ axis shows the error values and the right $y$-axis shows the standard deviation values. The comparison of the four different flow conditions is simplified by dividing the curve into the following four parts, which will be used subsequently:

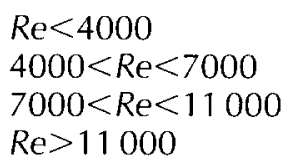

\section{The reference condition}

The unlinearized error curve for the reference condition (Figure 6a) is shown in Figure 7. For $R e<4000$ the ultrasonic flowmeter overestimates the flow velocity compared to the reference meter and for $4000<\operatorname{Re}<7000$ the error curve has a slope of $\approx-4 \cdot 10^{-4}(\% \operatorname{Re})$. The error is less than $\pm 0.1 \%$ for $7000<\operatorname{Re}<11000$ and for $\operatorname{Re}>11000$ the error drops to large negative values. The reason for this final drop is probably to be found in the increase of $\sigma_{1}$ and $\sigma_{2}$ indicating that the turbulent flow is distorting the ultrasonic pulse. The same increase of $\sigma_{1}$ and $\sigma_{2}$ can be found for $\operatorname{Re}<4000$, where the error becomes positive and rather large. This might be an effect of the nearness to the transition region causing instabilities in the flow.

\section{Straight pipe with a cone coupling in front of the meter}

Figure 8 shows the error curve when a cone coupling is put in front of the meter (as in Figure 6b). No significant difference can be traced when comparing the error curves in Figures 7 and 8 . It seems that a symmetrical disturbance does not affect the performance of the ultrasonic flowmeter.

\section{Single elbow in front of the meter body}

Figure 9 shows the error curve when a single elbow is put in front of the meter (as in Figure $6 \mathrm{c}$ ). For $\mathrm{Re}<4000$ the overestimation of the flow velocity reaches a lower maximum than in the reference condition, and for $4000<\operatorname{Re}<11000$ the error curve shows a consistent change of $-1 \%$. For $\operatorname{Re}>11000$ the slope of the underestimation is less than in the reference case. It seems that the velocity profile, caused by the elbow,

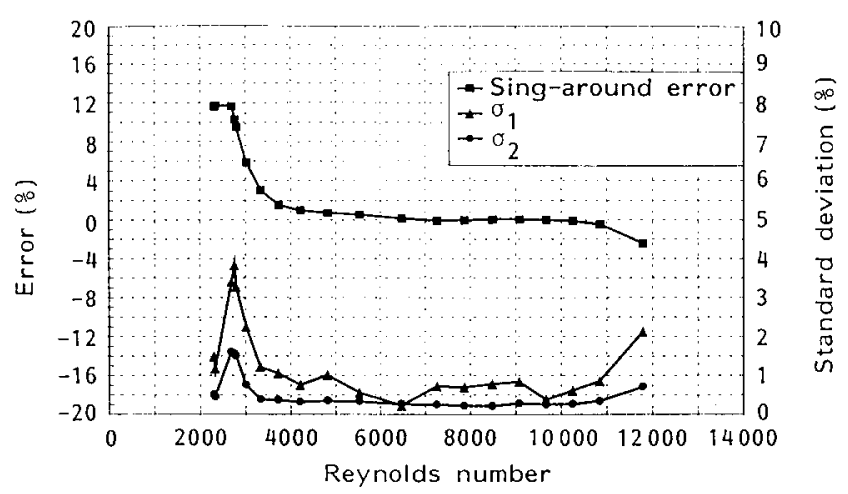

Figure 7 Ultrasonic flowmeter error as a function of Reynolds number for the flow caused by a straight $100 \mathrm{D}$ pipe 


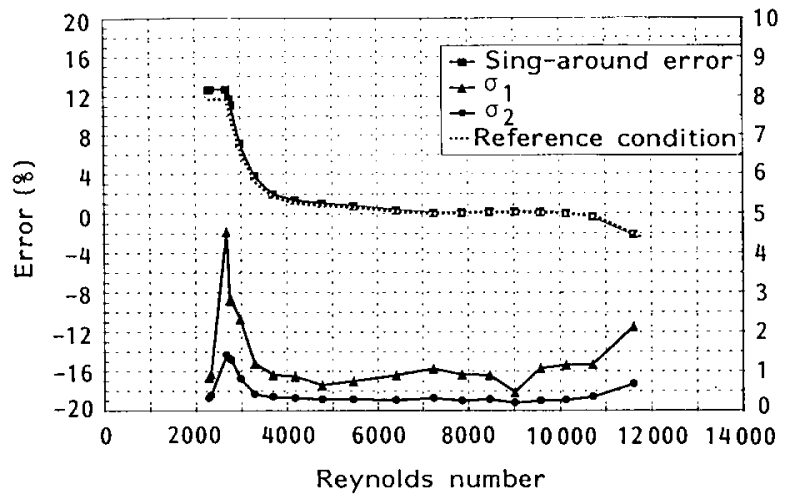

Figure 8 Ultrasonic flowmeter error as a function of Reynolds number for test condition 2 compared with the reference condition

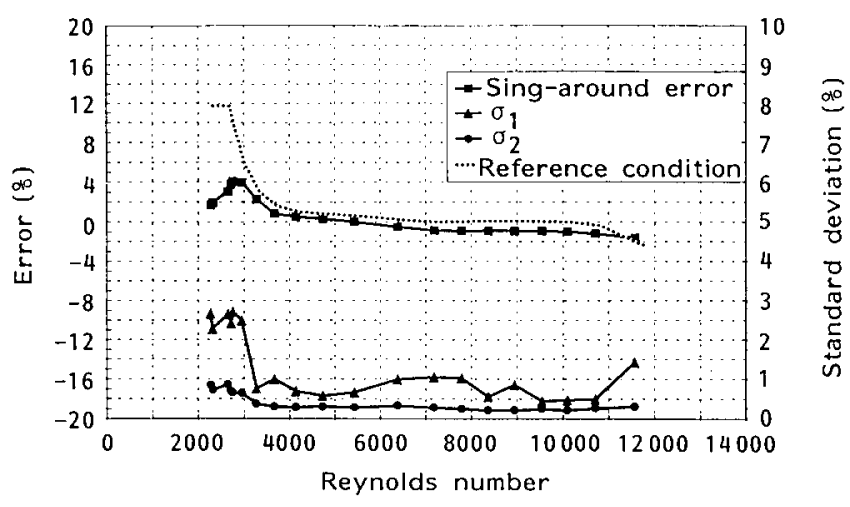

Figure 9 Error curve for a single elbow placed directly in front of the meter body with inlet angle $=0$. The error curve under the reference condition is shown as a dashed line

reduces both the overestimation and the underestimation for low and high Reynolds numbers respectively.

\section{Double elbow in front of the meter}

The result of a double elbow out of plane of the meter (Figure 6d) is shown in Figure 10. As in Figure 9 the overestimation for $\operatorname{Re}<4000$ reaches a lower maximum than in the reference condition. The slope for

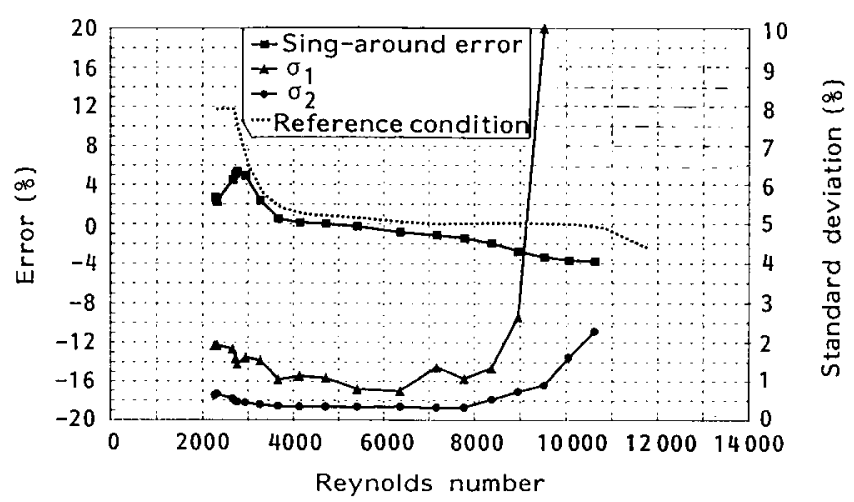

Figure 10 Error curve for a double elbow mounted $12 \mathrm{D}$ upstream. The error curve for the reference condition is shown as a dashed line
$4000<\operatorname{Re}<7000$ seems to be extended up to $\operatorname{Re} \approx$ 10600 , where the ultrasound is heavily distorted resulting in large negative errors. The distortion is indicated by the standard deviation values, $\sigma_{1}$ and $\sigma_{2}$, which, for $\operatorname{Re}>9000$, represent the heaviest turbulence for all configurations tested.

\section{Straight pipe between the single elbow and the meter body}

Figure 11 shows how the error changes when a straight pipe is mounted between the single elbow and the flowmeter. Four different pipe lengths, $L$, and four different flow rates were tested. The single elbow disturbance $(L=0)$ causes an underestimation of the flow velocity which changes into an overestimation for $L=10 D . L>40 D$ is enough to give reading errors within $\pm 1 \%$ compared to the reference condition.

\section{Straight pipe between the double elbow and the meter body}

Figure 12 shows how the error changes when a straight pipe is mounted between the double elbow and the flowmeter. Four different pipe lengths, $L$, and four different flow rates were tested. The double elbow disturbance $(L=0)$ causes an underestimation of the flow velocity which changes into an overestimation for $L<10 D$. However, for the double elbow configuration the length $L$ has to be increased to more than $80 \mathrm{D}$ (in the lower Reynolds number cases) in order to reestablish a reading error within $\pm 1 \%$ of the reference condition.

\section{Influence of sound path orientation}

For the single elbow the inlet angle is defined as the angle between the elbow plane and the transducer

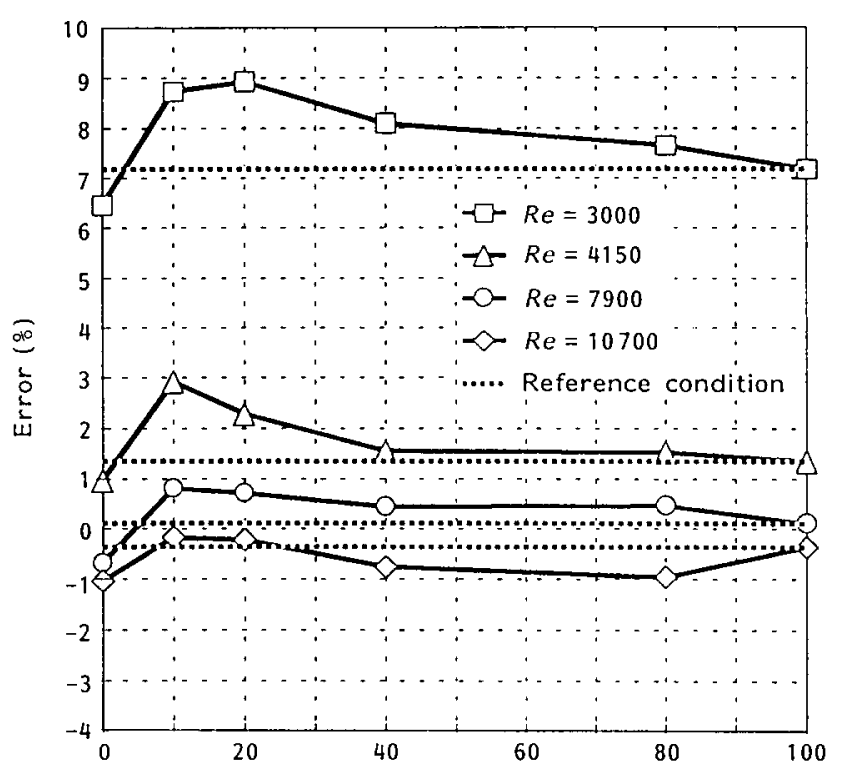

Distance between single elbow and flowmeter (pipe diameters)

Figure 11 Error curves for $10 \mathrm{D}, 20 \mathrm{D}, 40 \mathrm{D}$ and $80 \mathrm{D}$ straight piping between the single elbow and the meter. The error values for the reference condition are shown as dashed lines 


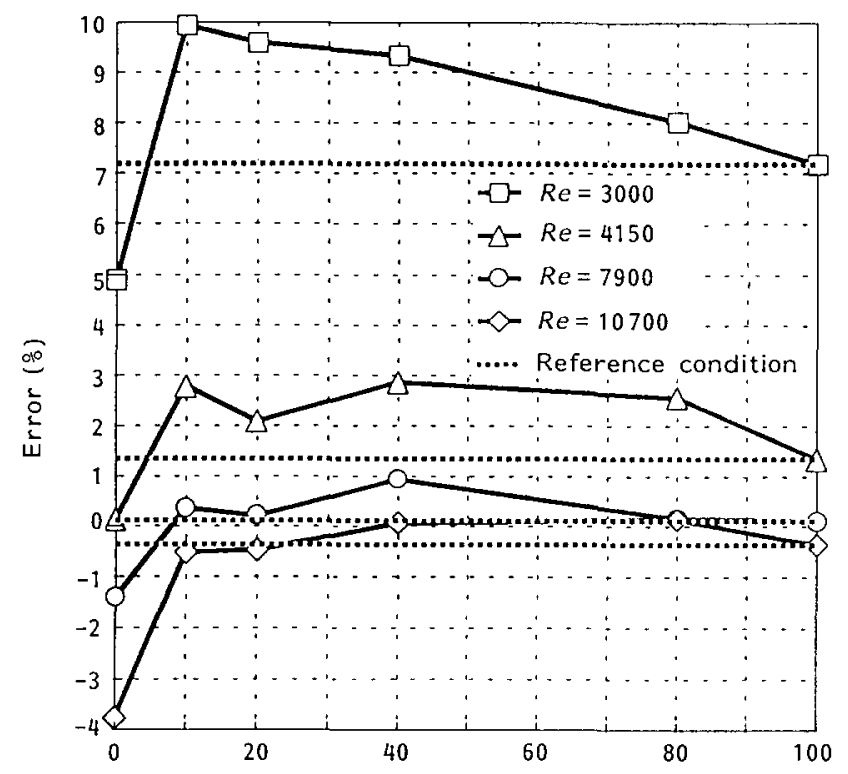

Distance between double elbow and flowmeter (pipe diameters)

Figure 12 Error curves for a double elbow mounted at different distances in front of the meter body. The error values for the reference condition are shown as dashed lines

plane (Figure 13). The change is made by rotating the pipework around the centre line of the flowmeter. Note that in all orientation tests, $L$ (in Figure 12) is zero.

Figure 14 shows the sing-around error as a function of the inlet angle for different Reynolds numbers. An interesting trend is revealed which is probably connected to the asymmetric flow profile caused by the single elbow. Changing the inlet angle from 0 to $315^{\circ}$ results in an error variation of up to $\pm 2 \%$ of the measured flow velocity.

For the out of plane double elbow the inlet angle is defined as the angle between the centre line of the straight pipe connected to the double elbow and the transducer plane (Figure 15). As in the single elbow case the angle is changed by rotating the pipework around the centre line of the flowmeter.

Figure 16 shows the sing-around error as a function of the inlet angle for different Reynolds numbers. Changing the inlet angle between 0 and $315^{\circ}$ results

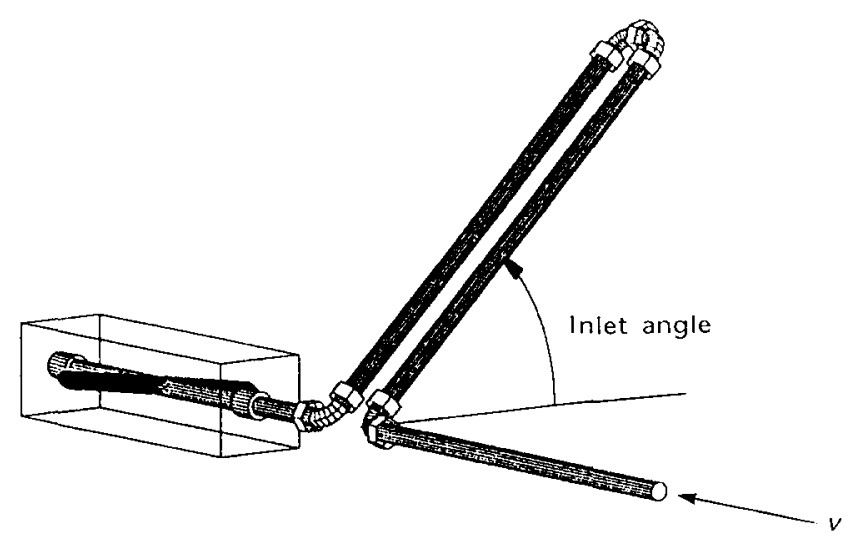

Figure 13 The inlet angle is changed by rotating the pipework around the centre line of the flowmeter

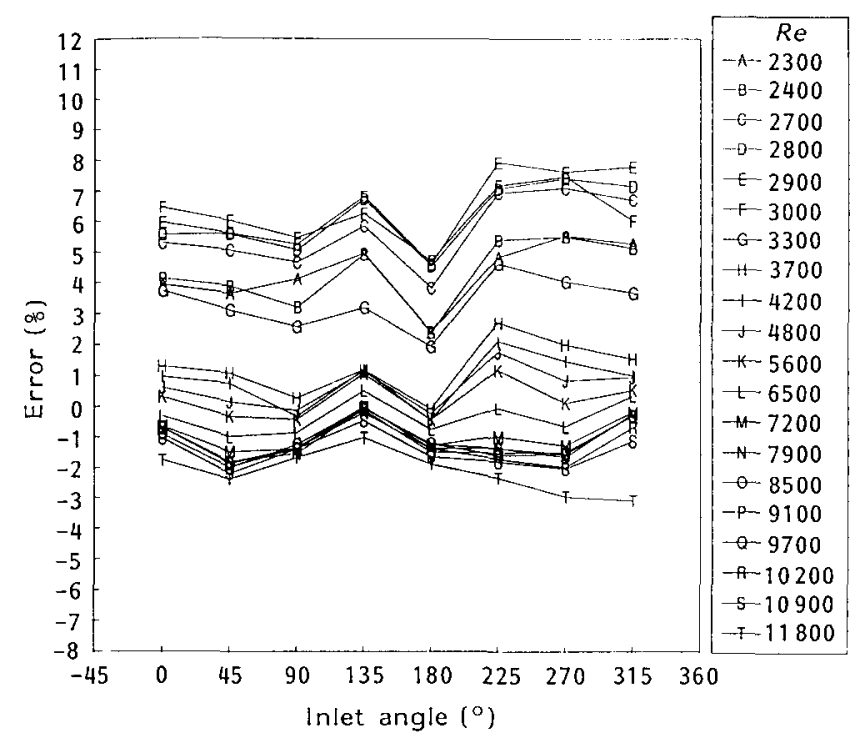

Figure 14 The sing-around error as a function of the inlet angle for the single elbow placed and rotated as shown in Figure 13

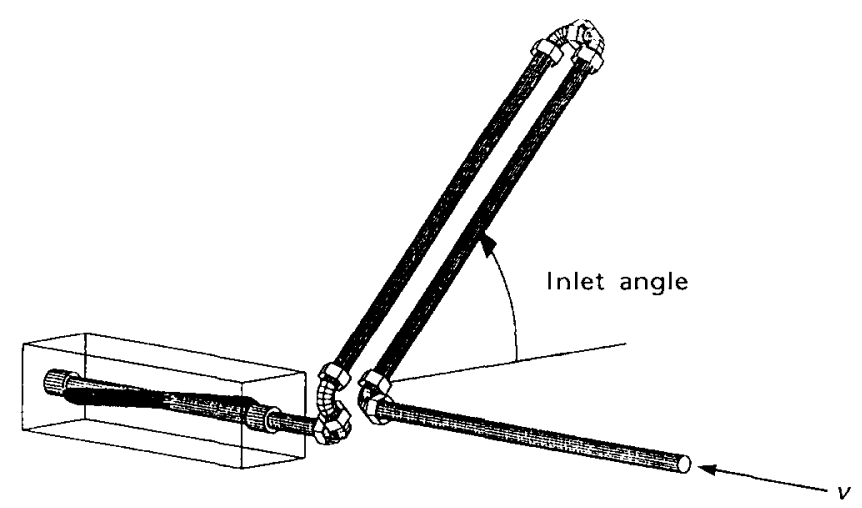

Figure 15 The inlet angle of the double elbow is changed by rotating the pipework around the centre line of the flowmeter

in an error variation of up to $\pm 3 \%$ of the measured flow velocity.

\section{Conclusions}

The measurement data presented here shows that the flow velocity value measured by the ultrasonic flowmeter is affected by the velocity distribution of the fluid between the ultrasonic transducers. For the slight symmetric disturbance produced by the cone coupling used between all pipe parts in the test-rig no significant change in the performance of the flowmeter occurs. The asymmetrical disturbances caused by the single and the double elbow used in our experiments generally cause an underestimation of the flow velocity.

Our measurements show that transitional flow $(R e<4000)$ results in an overestimation of the mean flow velocity. However, by introducing an asymmetrical disturbance, such as a single or double elbow in front of the meter, this overestimation is counteracted.

Adding a straight pipe between the single or double 


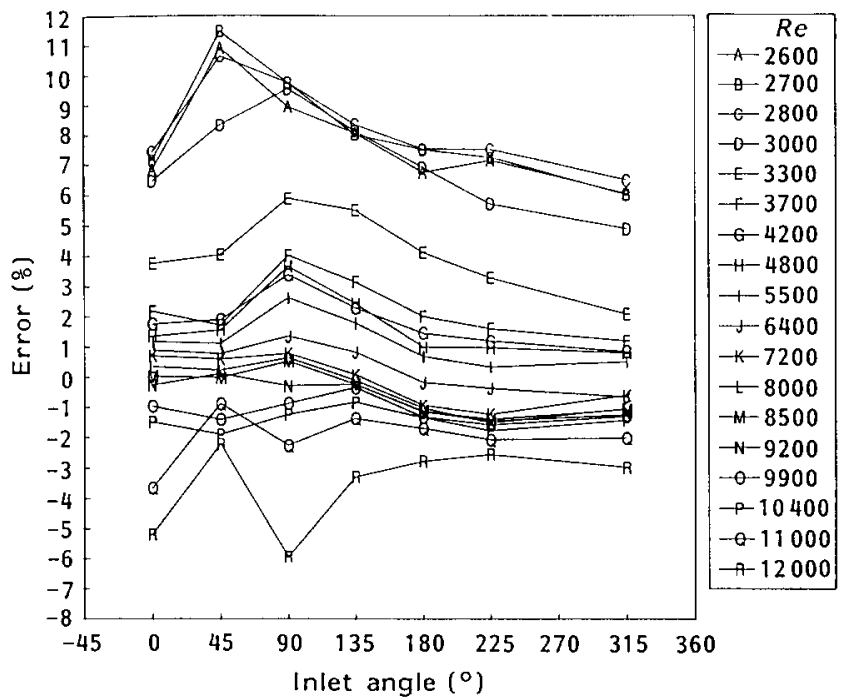

Figure 16 Sing-around error as a function of the inlet angle for the double elbow placed and rotated as shown in Figure 15

elbow disturbance and the flowmeter significantly changes the performance of the flowmeter. If a $10 \mathrm{D}$ straight pipe is put between the disturbance and the flowmeter the underestimation of the flow velocity is turned into an overestimation. Further increasing the pipe length decreases the overestimation and for the single elbow a $40 \mathrm{D}$ straight pipe between the disturbance and the meter body seems to be enough to give reading errors less than $\pm 1 \%$ relative to the reference condition. For the disturbance introduced by the double elbow an $80 \mathrm{D}$ pipe does not correct the flow profile enough to reduce the error to $\pm 1 \%$ relative to the reference condition.

Our measurements obviously show that the orientation of the sound path relative to the flow disturbance is of great importance. Errors of several percent are likely for certain orientations.
In conclusion, our measurements show that the geometry of the flowmeter including the position and width of the sound path will be of great importance in the design of an ultrasonic flowmeter that is independent of installation effects.

\section{Acknowledgement}

This work was funded by the Swedish National Board for Industrial and Technical Development.

\section{References}

1 Højholt, P. Installation effects on single and dual-beam ultrasonic flowmeters, International Conference on Flow Measurement in the mid 80s, NEL, Glasgow, Scotland (9-12 June 1986)

2 Heritage, J. E. The performance of transit time ultrasonic flowmeters under good and disturbed flow conditions. Flow Meas. Instrum. 1 (October 1989)

3 Luntta, E. and Halttunen, J. The effect of velocity profile on electromagnetic flow measurement, Department of Electrical Engineering Lab. of Measurement Technology, Tampere University of Technology, Finland (1988).

4 Halttunen, J. and Luntta, E. Effect of velocity profile on ultrasonic flowmeters, Department of Electrical Engineering Lab. of Measurement Technology, Tampere University of Technology, Finland (1989).

5 Halttunen, 1. and Luntta, E. Effect of single and double elbow out-of-plane configurations on the performance of ultrasonic flow meters, Tampere University of Technology, Laboratory of Measurement Technology, Tampere, Finland (1991)

6 Mattingly, G. E. and Yeh, T. T. Effects of pipe elbows and tube bundles on selected types of flowmeters, Fluid Flow Group, National Institute of Standards and Technology, Gaithersburg, Maryland, USA (1991)

7 Delsing, J. A new velocity algorithm for sing-around type flow meters IEEE Trans. UFFC 34(4) (July 1987)

8 Delsing, J. Ultrasonic gas flow meter with corrections for large dynamic metering range. Ultrasonics, 27 (November 1989)

9 Delsing, J. The zero flow performance of a sing-around type flow meter, 'Proc. IEEE Ultrasonics Symposium' (1990) 1541-1544

10 Delsing, J. and Håkansson, E. Absolute calibration of an ultrasonic gas flow meter. Project report, Lund Institute of Technology, LUTMDN/(TMVK-3144)/1-12/(1991)

11 'FT32 and Smaller Turbine Flow Meters Installation, Operation and Maintenance Manual, EG\&G Flow Technology (1989)

12 Youden, W. J. Graphical diagnosis of interlaboratory test results. 1. Ind. Qual. Control (May 1959) 24-28 